\title{
Nonlinearity of local dynamics promotes multi-chimeras
}

Cite as: Chaos 25, 083104 (2015); https://doi.org/10.1063/1.4927829

Submitted: 12 March 2015 . Accepted: 22 July 2015 . Published Online: 06 August 2015

Iryna Omelchenko, Anna Zakharova, Philipp Hövel, Julien Siebert, and Eckehard Schöll (D)
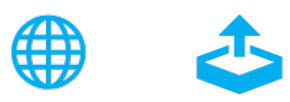

\section{ARTICLES YOU MAY BE INTERESTED IN}

Weak chimeras in minimal networks of coupled phase oscillators

Chaos: An Interdisciplinary Journal of Nonlinear Science 25, 013106 (2015); https: // doi.org/10.1063/1.4905197

\section{A classification scheme for chimera states}

Chaos: An Interdisciplinary Journal of Nonlinear Science 26, 094815 (2016); https:// doi.org/10.1063/1.4959804

\section{Synchronization of chaotic systems}

Chaos: An Interdisciplinary Journal of Nonlinear Science 25, 097611 (2015); https:// doi.org/10.1063/1.4917383

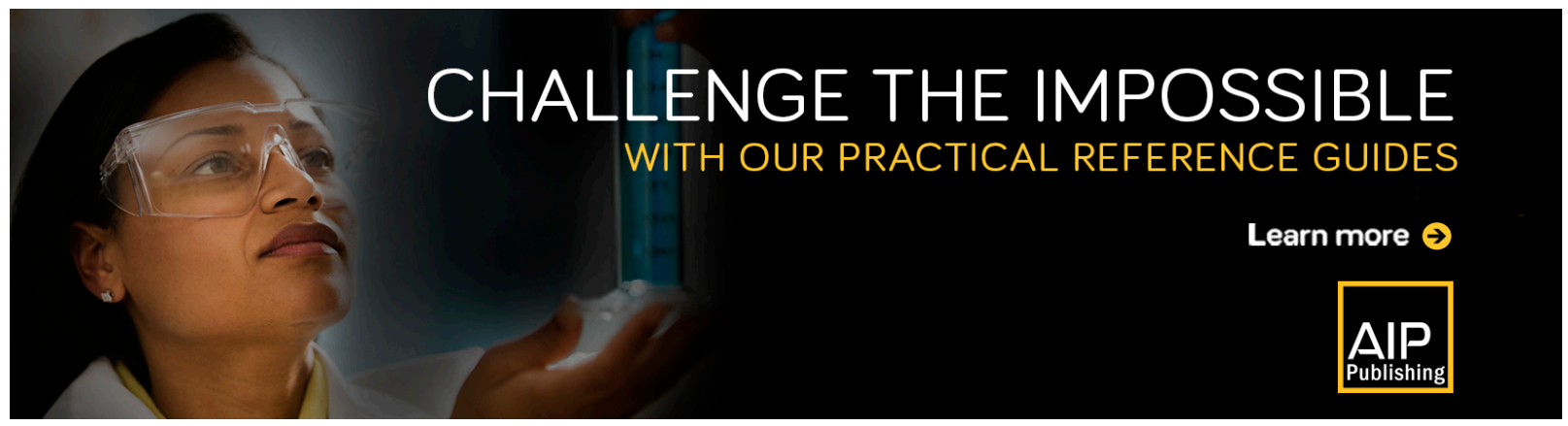




\title{
Nonlinearity of local dynamics promotes multi-chimeras
}

\author{
Iryna Omelchenko, ${ }^{1, a)}$ Anna Zakharova, ${ }^{1, b)}$ Philipp Hövel, , ,2,c) Julien Siebert,, d) \\ and Eckehard Schöll ${ }^{1, e)}$ \\ ${ }^{1}$ Institut für Theoretische Physik, Technische Universität Berlin, Hardenbergstraße 36, 10623 Berlin, Germany \\ ${ }^{2}$ Bernstein Center for Computational Neuroscience Berlin, Humboldt-Universität zu Berlin, Philippstraße 13, \\ 10115 Berlin, Germany
}

(Received 12 March 2015; accepted 22 July 2015; published online 6 August 2015)

\begin{abstract}
Chimera states are complex spatio-temporal patterns in which domains of synchronous and asynchronous dynamics coexist in coupled systems of oscillators. We examine how the character of the individual elements influences chimera states by studying networks of nonlocally coupled Van der Pol oscillators. Varying the bifurcation parameter of the Van der Pol system, we can interpolate between regular sinusoidal and strongly nonlinear relaxation oscillations and demonstrate that more pronounced nonlinearity induces multi-chimera states with multiple incoherent domains. We show that the stability regimes for multi-chimera states and the mean phase velocity profiles of the oscillators change significantly as the nonlinearity becomes stronger. Furthermore, we reveal the influence of time delay on chimera patterns. (C) 2015 AIP Publishing LLC.
\end{abstract}

[http://dx.doi.org/10.1063/1.4927829]

The investigation of coupled oscillatory systems is an important research field bridging between nonlinear dynamics, network science, and statistical physics, with a variety of applications in physics, biology, and technology. ${ }^{1,2}$ The analysis and numerical simulation of large networks with complex coupling schemes continue to open up new unexpected dynamical scenarios. Chimera states are an example for such intriguing phenomena; they exhibit a hybrid structure combining coexisting domains of both coherent (synchronized) and incoherent (desynchronized) dynamics and were first reported for the well-known model of phase oscillators. ${ }^{3,4}$ In this paper, we investigate the influence of the local dynamics of the oscillators upon the resulting chimera patterns. Using the Van der Pol oscillator, which is a model allowing for a continuous transition between sinusoidal and strongly nonlinear relaxation oscillations by tuning a single parameter, we show that multi-chimera patterns with multiple incoherent domains are promoted by increasing the nonlinearity of the local oscillator dynamics.

\section{INTRODUCTION}

The last decade has seen an increasing interest in chimera states in dynamical networks. ${ }^{5-13}$ It was shown that they are not limited to phase oscillators but can be found in a large variety of different systems including time-discrete maps, ${ }^{14}$ time-continuous chaotic models, ${ }^{15}$ neural systems, ${ }^{16-18}$ and Boolean networks. ${ }^{19}$ Moreover, chimera states were found in systems with higher spatial dimensions. ${ }^{7,9,13,20-22}$ Together with the initially reported chimera

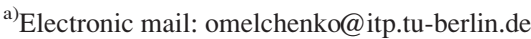

b)Electronic mail: anna.zakharova@tu-berlin.de

${ }^{c)}$ Electronic mail: phoevel@physik.tu-berlin.de

d)Electronic mail: j.siebert@mailbox.tu-berlin.de

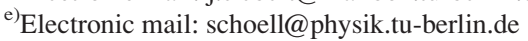

states, which consist of one coherent and one incoherent domain, new types of these peculiar states having multiple incoherent regions, ${ }^{16,18,23-25}$ as well as amplitude-mediated, ${ }^{26,27}$ and pure amplitude chimera and chimera death states $^{28}$ were discovered.

In many systems, the form of the coupling defines the possibility to obtain chimera states. The nonlocal coupling has generally been assumed to be a necessary condition for chimera states to evolve in coupled systems. However, recent studies have shown that even global all-to-all coupling ${ }^{27,29-31}$ and more complex coupling topologies allow for the existence of chimera states. ${ }^{32-37}$ Furthermore, timevarying network structures can give rise to alternating chimera states. ${ }^{38}$

The important question of the main features that give rise to chimera states in coupled systems has been widely discussed, but no conclusive answer has been given yet. In systems of phase oscillators, the value of the phase lag parameter $\alpha$, which occurs in the coupling function, is crucial. In nonlocally coupled systems, the range of the coupling and its strength play the key role. If the local dynamics of each unit is described by a two- or higher dimensional system, then the interaction scheme between the units plays an important role, i.e., which variable is coupled to which variable of the other nodes. Chimera states have also been shown to be robust against inhomogeneities of the local dynamics and coupling topology. ${ }^{37,39}$

Possible applications of chimera states in natural and technological systems include the phenomenon of unihemispheric sleep, ${ }^{40}$ bump states in neural systems, ${ }^{41,42}$ power grids, ${ }^{43}$ or social systems. ${ }^{44}$ Many works considering chimera states have mostly been based on numerical results. A deeper bifurcation analysis ${ }^{45}$ and even a possibility to control chimera states ${ }^{46,47}$ were obtained only recently.

The experimental verification of chimera states was first demonstrated in optical $^{48}$ and chemical ${ }^{49,50}$ systems. Further 
experiments involved mechanical, ${ }^{51}$ electronic ${ }^{52,53}$ and electrochemical ${ }^{54,55}$ oscillator systems, Boolean networks, ${ }^{19}$ the optical comb generated by a passively mode-locked quantum dot laser, ${ }^{56}$ and superconducting quantum interference devices. ${ }^{57}$

In previous investigations of chimera states, usually the character of the local node dynamics has been considered as fixed. In the current study, we address the issue of the impact of the local dynamics. We analyze the properties of chimera states, when the dynamics of individual oscillators smoothly changes from sinusoidal to nonlinear relaxation oscillations. For this reason, we choose the Van der Pol oscillator to describe the dynamics of each node. The Van der Pol oscilla$\operatorname{tor}^{58}$ has a long history of being used in both the physical and biological sciences, as a generic model for electrical circuits $^{59}$ and action potentials of neurons, respectively.

\section{THE MODEL}

In our study, we consider a system of nonlocally coupled Van der Pol oscillators with ring topology, where each element of the system interacts with a fixed range of its neighbors in both directions

$$
\begin{aligned}
\ddot{u}_{k}= & \varepsilon\left(1-u_{k}^{2}\right) \dot{u}_{k}-u_{k} \\
& +\frac{\sigma}{2 R} \sum_{j=k-R}^{k+R}\left[b_{1}\left(u_{j}-u_{k}\right)+b_{2}\left(\dot{u}_{j}-\dot{u}_{k}\right)\right],
\end{aligned}
$$

with $k=1, \ldots, N$ where all indices are taken modulo $N, \varepsilon$ is the bifurcation parameter of the individual oscillator, $\sigma$ denotes the strength of the coupling, $R$ is the number of coupled neighbors (in each direction), and $b_{1}, b_{2}$ are the interaction parameters. For such a form of coupling, it is convenient to consider the ratio $r=R / N$, which we denote as a coupling range. The uncoupled Van der Pol oscillator has a stable trivial steady state $u=0$ for $\varepsilon<0$ and exhibits a supercritical Hopf bifurcation at $\varepsilon=0$. Here, we consider $\varepsilon>0$.

Introducing a new variable $v_{k}=\dot{u}_{k}$, Eq. (1) can be rewritten in the form of a two-dimensional system

$$
\begin{aligned}
\dot{u}_{k}= & v_{k} \\
\dot{v}_{k}= & \varepsilon\left(1-u_{k}^{2}\right) v_{k}-u_{k} \\
& +\frac{\sigma}{2 R} \sum_{j=k-R}^{k+R}\left[b_{1}\left(u_{j}-u_{k}\right)+b_{2}\left(v_{j}-v_{k}\right)\right] .
\end{aligned}
$$

The form of the coupling in the system Eq. (1) or (2) is inspired from biological systems, describing interaction of the cells or pattern generation in locomotion. ${ }^{60,61} \mathrm{~A}$ similar form of the coupling is also used in mechanics. ${ }^{62}$ The crosscouplings between the $u$ - and the $v$-variable play an important role, and they were shown to be necessary for the existence of chimera and multi-chimera states in systems of nonlocally coupled FitzHugh-Nagumo oscillators. ${ }^{16}$

The dynamics of the system Eq. (2) is determined by five parameters: $\varepsilon$ defines the dynamics of each individual unit, and the parameters $\sigma, R, b_{1}$, and $b_{2}$ specify the coupling.
In order to find suitable values for some of the system parameters in the regime where Eq. (2) can describe chimera states, we will use the experience from simpler systems of coupled Kuramoto phase oscillators. For this reason, we transform our system using the phase averaging technique on a rotating frame for slowly varying amplitude $r_{k}$ and phase $\theta_{k}: u_{k}(t)=$ $r_{k}(t) \sin \left(t+\theta_{k}(t)\right)$ and $v_{k}(t)=r_{k}(t) \cos \left(t+\theta_{k}(t)\right)$. As a result, we obtain the approximate system

$$
\begin{aligned}
\dot{r}_{k}= & \frac{\varepsilon}{8} r_{k}\left[\left(4-\frac{2 \sigma}{\varepsilon R}(2 R+1) b_{2}\right)-r_{k}^{2}\right] \\
& +\frac{\sigma}{4 R} \sqrt{b_{1}^{2}+b_{2}^{2}} \sum_{j=k-R}^{k+R} r_{j} \cos \left(\theta_{k}-\theta_{j}+\alpha\right) \\
\dot{\theta}_{k}= & \frac{\sigma}{4 R}(2 R+1) b_{1} \\
& -\frac{\sigma}{4 R} \sqrt{b_{1}^{2}+b_{2}^{2}} \sum_{j=k-R}^{k+R} \frac{r_{j}}{r_{k}} \sin \left(\theta_{k}-\theta_{j}+\alpha\right)
\end{aligned}
$$

with $\alpha=\arctan \left(b_{1} / b_{2}\right), b_{2}>0$ and $k=1, \ldots, N$.

The parameter $\alpha$ in the system (3) can be associated with the phase lag parameter in the systems of coupled phase oscillators. ${ }^{4}$ This parameter is crucial for the appearance of chimera states in the phase oscillator network. In Ref. 8, it was shown that a value of the phase lag parameter close to but slightly less than $\pi / 2$ allows for the existence of chimera states.

In the following, using the experience from the phase oscillator network, we fix the interaction parameters to be $b_{1}=1$ and $b_{2}=0.1$, such that $\alpha \approx 1.47$ is close to $\pi / 2$. With this parameter choice, we will focus further on the original system Eq. (2) and vary the parameter $\varepsilon$ that defines the type of local dynamics of each element, as well as the coupling parameters $\sigma$ and $r$ describing the strength and the range of the coupling, respectively.

\section{THE IMPACT OF LOCAL DYNAMICS}

Varying the bifurcation parameter $\varepsilon$ results in a change of the character of the local node dynamics. If $\varepsilon$ is small, the uncoupled individual elements of the system perform harmonic oscillations on a limit cycle, which is approximately a circle. With increasing $\varepsilon$, the individual limit cycle becomes distorted and changes its form to relaxation oscillations.

Figure 1 demonstrates examples of chimera states for the system of $N=1000$ elements, $\varepsilon=0.2$, and decreasing coupling range. The upper panels depict snapshots of the variables $u_{k}$ for fixed time $T=50000$. As initial conditions, we use randomly distributed phases on the circle $u^{2}+v^{2}=4$, i.e., around the limit cycle of the uncoupled system, which is approximately a circle of radius 2 . One can clearly distinguish coherent and incoherent domains, a characteristic signature of chimera states. Elements that belong to the incoherent domain are scattered along the limit cycle, as shown with red points in the bottom panels of Fig. 1, where the black line denotes the limit cycle of the uncoupled unit with corresponding value $\varepsilon=0.2$. The individual nodes perform a nonuniform rotational motion, but neighboring oscillators are not phase-locked. To illustrate this, the middle 
(a)
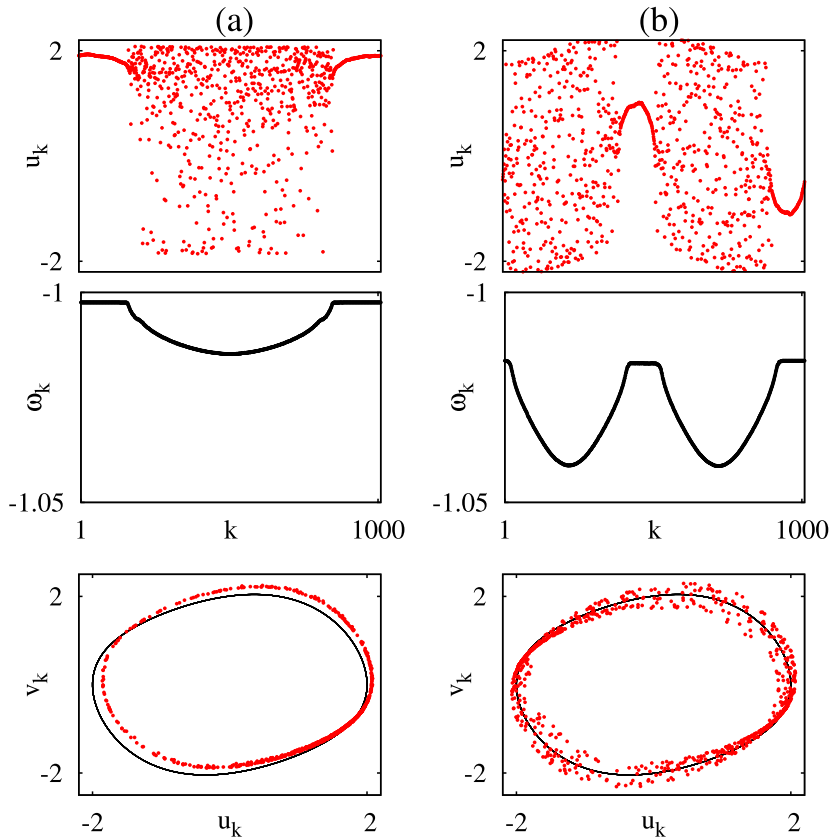

(c)
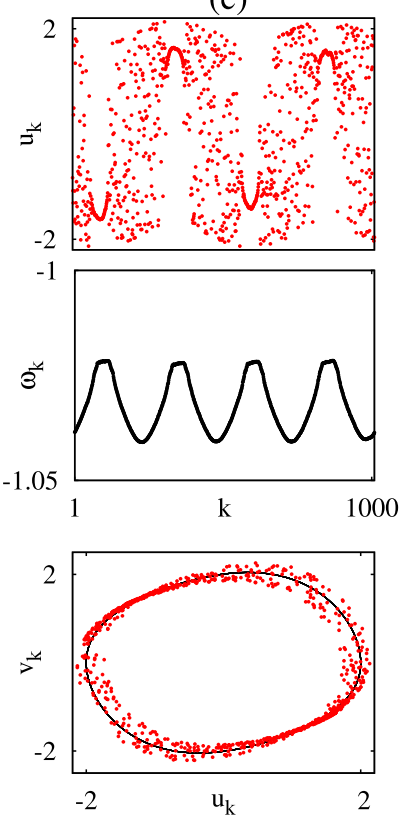

(d)
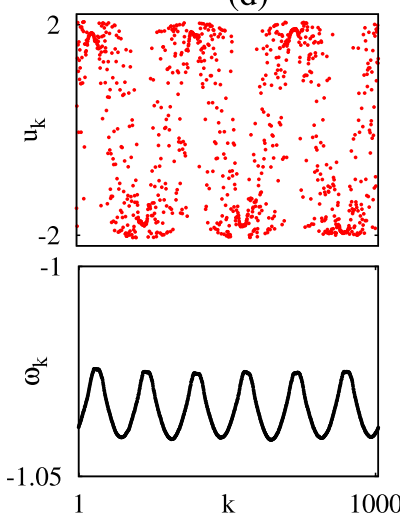

FIG. 1. Snapshots of the variables $u_{k}$ (upper panels), mean phase velocities $\omega_{k}$ (middle panels), and snapshots in the phase space ( $u_{k}$, $v_{k}$ ) (bottom panels, limit cycle of the uncoupled unit shown black). (a) $r=0.35, \sigma=0.05$, (b) $r=0.2, \sigma=0.09$, (c) $r=0.13, \sigma=0.09$, (d) $r=0.1, \sigma=0.09$. Other parameters: $N=1000, b_{1}=1, b_{2}=0.1$, and $\varepsilon=0.2$.

panels of Fig. 1 show the mean phase velocities for each oscillator calculated as $\omega_{k}=2 \pi M_{k} / \Delta T, k=1, \ldots, N$, where $M_{k}$ is the number of complete rotations around the origin performed by the $k$-th node during the time interval $\Delta T$. Throughout the paper, we use $\Delta T=50000$ for the calculation of the mean phase velocities $\omega_{k}$, corresponding to several thousand periods. The values of $\omega_{k}$ lie on a continuous curve and the interval of constant $\omega_{k}$ corresponds to the coherent domain, where neighboring elements are phaselocked. This mean phase velocity profile is a clear indication of chimera states and similar to the case of coupled Kuramoto phase oscillators. ${ }^{3,4}$

In addition to chimera states with one incoherent domain [Fig. 1(a)], we observe chimera states with multiple incoherent domains shown in Figs. 1(b)-1(d), i.e., multi-chimera states. In the following, we will use the notation $n$-chimera for a chimera state with $n$ coherent and $n$ incoherent domains. In analogy with networks of phase oscillators, here we observe chimera states where the number of incoherent domains is even. The number of incoherent domains increases with decreasing coupling range.

Figure 2(a) shows the stability regimes for chimera states with one and multiple incoherent domains in the plane of coupling range $r$ and coupling strength $\sigma$ for $\varepsilon=0.2$.
Indeed, for large coupling range, we observe the stability regime for chimera states with one incoherent domain, and regimes for chimera states with two, four, and six incoherent domains follow subsequently with decreasing coupling range. The overlaps of these regimes are characterized by multistability, when each of the chimera states can be obtained in the system depending on the choice of the initial conditions. The regimes shown in the diagram are obtained by starting from the chimera states shown in Fig. 1, and using this pattern as initial condition for the neighboring parameter set, and so forth with a step size of $\Delta r=0.01$ and $\Delta \sigma=0.01$. Black squares denoted by A-D show values of the parameters $(r, \sigma)$ that correspond to the examples presented in Figs. 1(a)-1(d), respectively.

For larger coupling strength $\sigma$, we observe coherent states in the system (2). They are characterized by the wavenumber $K$ defining the number of maxima (minima) in the spatial profile, and $K=0$ corresponds to complete in-phase synchronization. The wavenumber increases with decreasing coupling range, and exemplary snapshots are shown in the insets of Fig. 2(a). For large coupling strength $\sigma$, the system is characterized by high multistability, and depending upon initial conditions, one can obtain coherent solutions with different wavenumbers. In our system, there exist two different
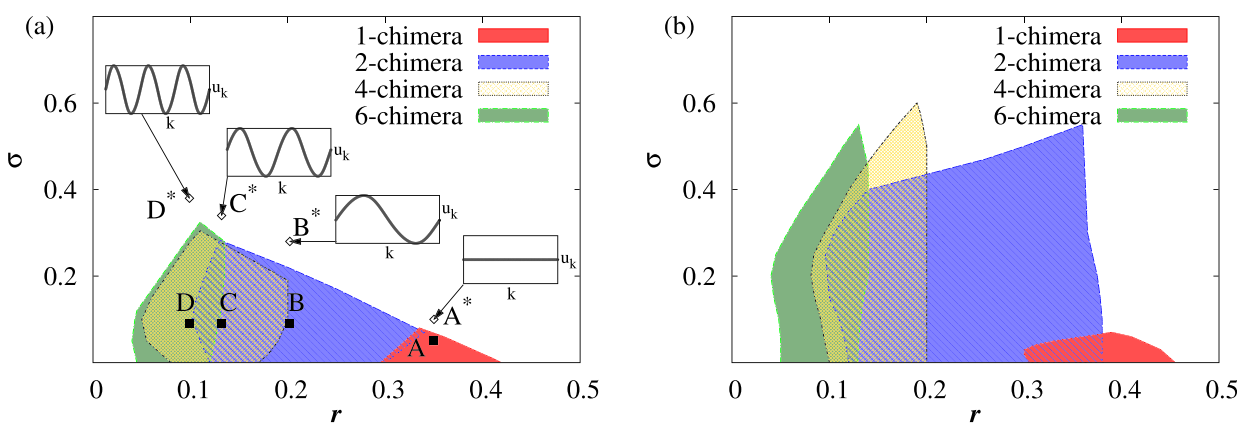

FIG. 2. Stability regimes for multiple chimera states. (a) $\varepsilon=0.2$, black squares marked by A-D show parameter values corresponding to panels (a)-(d) in Fig. 1. The insets show snapshots of coherent spatial profiles for parameter values $A^{*}$ $(r=0.35, \sigma=0.12 ; K=0), B^{*}(r=0.2$, $\sigma=0.28 ; K=1), C^{*}(r=0.13, \sigma=0.34$; $K=2), D^{*}(r=0.1, \sigma=0.38 ; K=3)$; (b) $\varepsilon=0.4$. Other parameters as in Fig. 1 . 
types of chimeras, amplitude-mediated chimeras and pure phase chimeras, and these are generated by different bifurcation mechanisms. The amplitude-mediated 2-, 4-, 6-chimeras (Fig. 2(a), snapshots in Figs. 1(b)-1(d), top panel) are generated from smooth, completely coherent spatial profiles of wavenumbers $K=1,2,3$, respectively, by a coherenceincoherence bifurcation with decreasing coupling strength as indicated by the insets in Fig. 2(a). At the onset of chimeras, the smooth coherent profiles break up into spatially coherent domains corresponding to the upper and the lower parts of these profiles and incoherent domains in between. Therefore, these incoherent domains occur in pairs (2-, 4-, 6-chimeras). Such coherence-incoherence bifurcations have also been observed for other local dynamics, e.g., logistic maps and Rössler systems, ${ }^{14}$ the cosine map, ${ }^{48}$ and Stuart-Landau oscillators. ${ }^{28}$ In contrast, the pure phase chimeras (1-chimera in Figs. 2(a) and 2(b)) arise from completely in-phase synchronized $(K=0)$ profiles. The shift of the regimes for (multiple) $n$-chimeras to smaller coupling range $r$ with increasing $n$ is typical for various nonlocally coupled systems. ${ }^{14-16,28,48}$

For small $\varepsilon$, the limit cycle of each individual Van der Pol oscillator is close to a circle, corresponding to sinusoidal oscillations, and the similarities to the chimera states in a system of phase oscillators are clearly revealed. However, the hybrid solutions we observe in the system of coupled Van der Pol oscillators demonstrate chimera behavior both for phases and amplitudes. This can be seen in the bottom panels of Fig. 1, where red dots denoting the snapshot of all nodes deviate in their amplitudes slightly from the limit cycle of the uncoupled unit (black line).

Figure 2(b) depicts the stability regimes for chimera states in the system (2) with $\varepsilon=0.4$. Compared to the case of $\varepsilon=0.2$, the regimes for the chimera states with multiple incoherent domains become larger, and chimera states in this case can be obtained for a wider range of coupling strength $\sigma$. The reason why the regions in Fig. 2(b) $(\varepsilon=0.4)$ are larger than those in Fig. 2(a) $(\varepsilon=0.2)$ is related to the following qualitative argument: If the coefficient $\varepsilon$ of the nonlinear term in Eq. (1) is increased, the coefficient $\sigma$ of the coupling term has to be scaled up accordingly to balance the nonlinear term. Hence, as $\varepsilon$ is increased, the chimera regions extend to larger values of $\sigma$.

When the parameter $\varepsilon$ is increased, the limit cycle of the individual uncoupled Van der Pol oscillators deforms, and the dynamics on the cycle becomes slow-fast type. Further increasing $\varepsilon$ leads to strongly nonlinear relaxation oscillations. This will be discussed in the following.
Figure 3(a) depicts the stability regimes for system (2) with $\varepsilon=0.8$. Compared to the cases of smaller $\varepsilon$, there are several qualitative differences in the stability regimes of chimera states. First, chimera states can be observed for a much larger range of coupling strength $\sigma$. Second, chimera states with one incoherent part cannot be observed in the system any more: for large coupling range, we observe chimera states with four incoherent parts, and furthermore with decreasing coupling range, the multiplicity of the incoherent domains of the chimera states increases. Black triangles denoted by E, F, and $\mathrm{G}$ show values for the parameter pairs $(r, \sigma)$ that correspond to examples of chimera states depicted in Figs. 4(a)-4(c), respectively.

The peculiarity of the diagram presented in Fig. 3(a) is the presence of two separate regimes for chimera states with four incoherent domains. Analyzing this diagram in more detail, one can see that there are two qualitatively different regions. The first region appears for large coupling strengths and contains stability regimes for chimera states with two and four incoherent domains (yellow region containing point $\mathrm{E}$ and blue region). These states can be characterized by strong amplitude dynamics, and the maximum values of the mean phase velocity profile correspond to the coherent domains of chimera states. The example presented in Fig. 4(a) [corresponding to point $\mathrm{E}$ in Fig. 3(a)] depicts these features. Compared with Fig. 1 for small $\varepsilon$, we notice that the chimera states shown there also show distinct variations along the limit cycle, and the coherent domains of the chimera states correspond to the maximum in the mean phase velocity profiles.

The second, qualitatively different part of the diagram in Fig. 3(a), includes three regions for small coupling strengths (yellow including point $\mathrm{F}$, green, and gray). These regions form a similar sequence with increasing multiplicity of the chimera starting from four incoherent parts. However, they exhibit a qualitative difference. Inspecting Figs. 4(b) and 4(c), which show examples that correspond to the parameter pairs $(r, \sigma)$ denoted by $\mathrm{F}$ and $\mathrm{G}$, one can notice that the amplitude dynamics becomes weaker in these cases, and the network solution is close to the limit cycle of the uncoupled node shown as black line in the bottom panels. Moreover, the minimum of the mean phase velocities profiles now corresponds to the coherent domains of the chimera states.

The difference between Fig. 4(a) on one hand and Figs. 4(b) and 4(c) on the other hand is due to two different types of chimeras. The 2-, 4-, 6-chimeras in Figs. 2(a), 2(b) and Fig. 3(a) (point E), and the chimeras in Figs. 3(F,G,H,I,J) belong to two different types of chimeras: Figs. 2(a), 2(b)
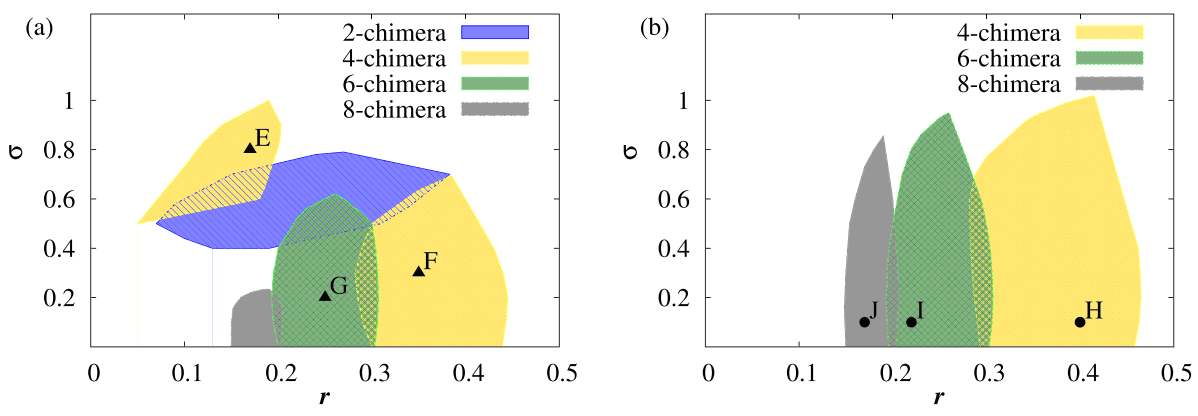

FIG. 3. Stability regimes for multiple chimera states. (a) $\varepsilon=0.8$, black triangles marked by E-G show parameter values corresponding to the panels (a)(c) in Fig. 4; (b) $\varepsilon=1.5$, black circles marked by $\mathrm{H}-\mathrm{J}$ denote parameter values corresponding to the panels (a)-(c) in Fig. 5. Other parameters as in Fig. 1. 
(a)
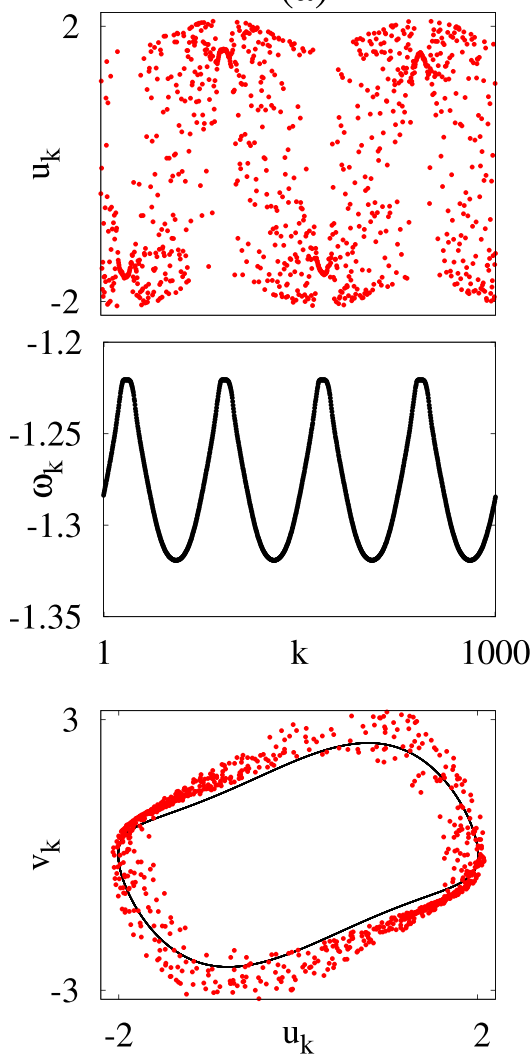

(b)
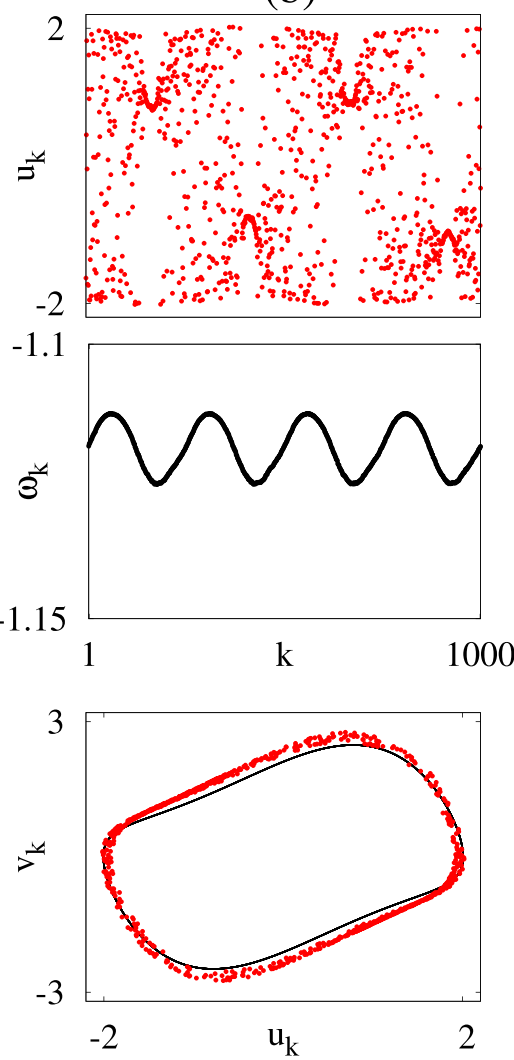

(c)

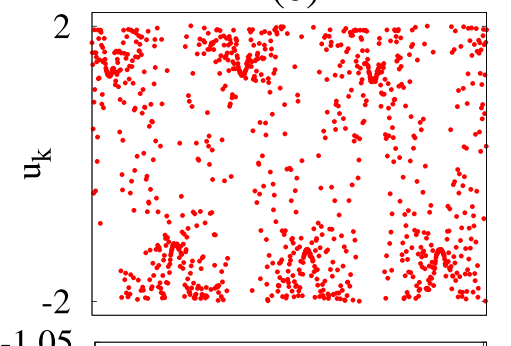

$-1.05$
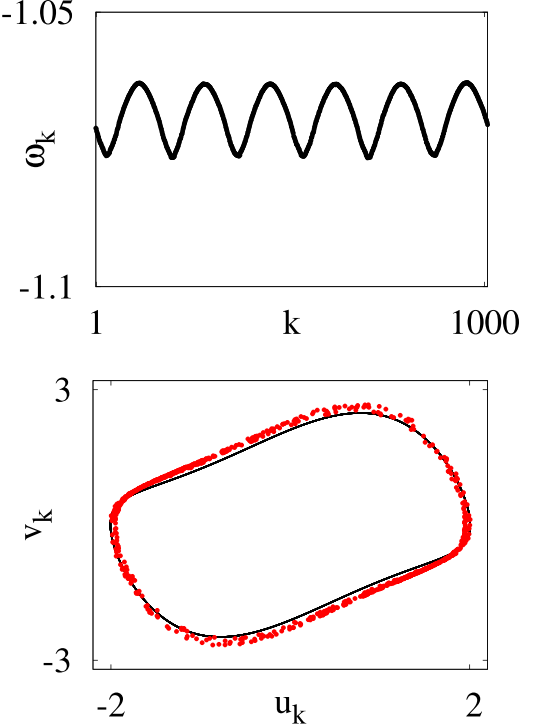

FIG. 4. Snapshots of the variables $u_{k}$ (upper panels), mean phase velocities $\omega_{k}$ (middle panels), and snapshots in the phase space ( $u_{k}$, $v_{k}$ ) (bottom panels, limit cycle of the uncoupled unit shown black). (a) $r=0.17, \sigma=0.8$, (b) $r=0.35, \sigma=0.3$, (c) $r=0.25, \sigma=0.2$. Other parameters as in Fig. 3(a).

and Fig. 3(a), point E (corresponding to phase portraits shown in Figs. 1(b)-1(d) and Fig. 4(a)) correspond to amplitude-mediated chimeras with strong amplitude-phase coupling, whereas Fig. 3(a), points F, G and Fig. 3(b) (corresponding to phase portraits shown in Figs. 4(b), 4(c) and Figs. 5(a)-5(c)) correspond to pure phase chimeras similar to the ones found for Kuramoto phase oscillators, and since the phase oscillator model can generally be obtained from amplitude-phase models in the weak coupling limit, they occur in the stability diagram (Fig. 3(a)) only for small coupling strength (points F,G), as opposed to the amplitudemediated chimeras (point E). This difference is visible in the phase portraits of Figs. 1, 4, 5 (bottom panels), where the spread of the various oscillators around the cycle of the uncoupled oscillator (black cycle) is large for amplitudemediated chimeras and very small for pure phase chimeras where the phase of the cycle is the only dynamical degree of freedom. The difference also shows up in the smaller amplitude variation of the mean phase velocity in the middle panels of Figs. 4(b) and 4(c) (pure phase chimeras) as compared to Fig. 4(a) and in the inverted $\omega_{k}$ profiles: the coherent regions correspond to the minima (Figs. 4(b) and 4(c)) and maxima (Fig. 4(a)), respectively.

Further increase of the parameter $\varepsilon$ of individual Van der Pol oscillators leads to an even stronger deformation of the limit cycle. In the $(r, \sigma)$ parameter plane, the stability regimes for chimera states with four and more incoherent domains can be observed as shown in Fig. 3(b) for $\varepsilon=1.5$. The effect of the coexistence of two qualitatively different types of chimera states is not present there any more, in contrast to the case of $\varepsilon=0.8$. Only the second type of the chimera states is observed in the systems now, and the stability regimes are enlarged towards larger coupling strengths.

Figure 5 depicts examples of multi-chimera states that correspond to the parameter pairs $(r, \sigma)$ denoted by $\mathrm{H}$, I, and $\mathrm{J}$ in Fig. 3(b). The coherent domains of the chimera states correspond to the minimum of the mean phase velocity profile, and all oscillators stay very close to the limit cycle of the single uncoupled unit, thus the amplitude dynamics of the chimera states in the systems with large $\varepsilon$ is not as pronounced as in the networks with small $\varepsilon$.

We conclude that the nonlinearity of the local dynamics indeed strongly influences chimera states in system (2). The character of the amplitude dynamics, the frequencies of the oscillators belonging to the coherent and incoherent domains of the chimera states, i.e., the mean phase velocity profiles, and the stability regimes in the coupling parameter plane undergo a qualitative change with variation of the parameter $\varepsilon$. Stronger nonlinearity (larger $\varepsilon$ ) results in the dominance of multi-chimera states with weak amplitude dynamics.

\section{TIME-DELAYED COUPLING}

Together with the character of the local dynamics, the coupling between the individual units plays an important role for the properties of the chimera states. Time-delayed coupling if compared to the instantaneous one represents a more realistic way to model the interaction between the 
(a)
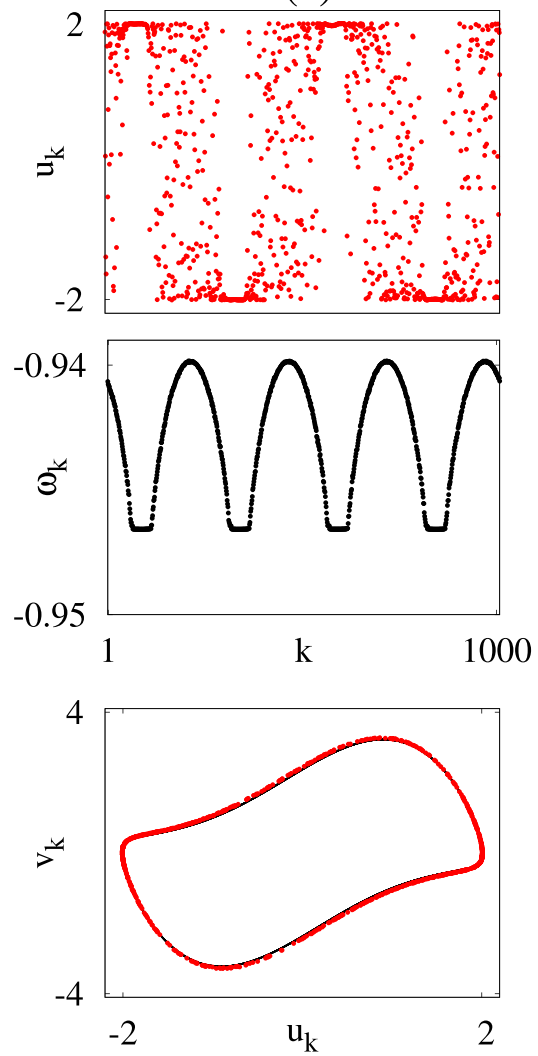

(b)

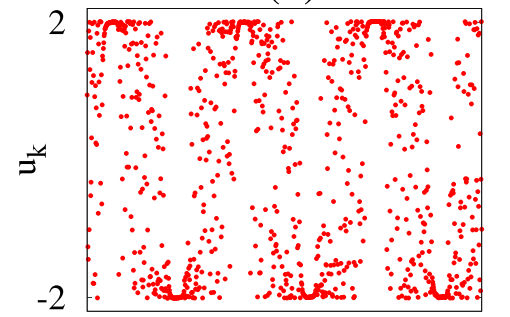

$-0.94$
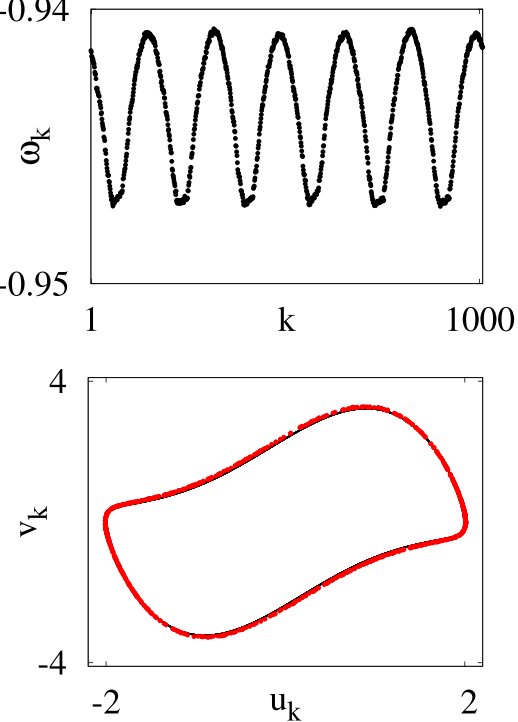

(c)

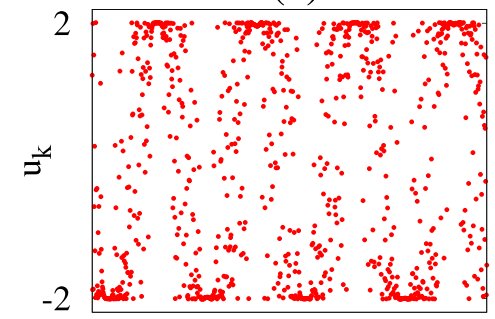

$-0.94$
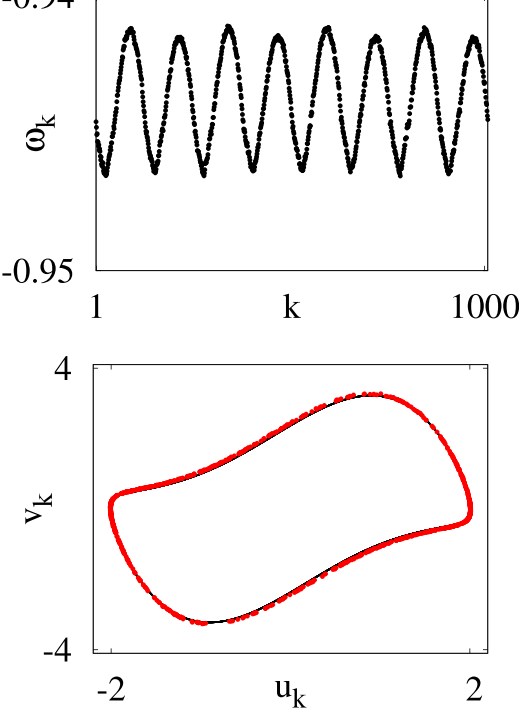

FIG. 5. Snapshots of the variables $u_{k}$ (upper panels), mean phase velocities $\omega_{k}$ (middle panels), and snapshots in the phase space ( $u_{k}$, $v_{k}$ ) (bottom panels, limit cycle of the uncoupled unit shown black). (a) $r=0.4, \sigma=0.1$, (b) $r=0.22, \sigma=0.1$, (c) $r=0.17, \sigma=0.1$. Other parameters as in Fig. 3(b).

coupled units. Usually, the coupling range and strength influence the multiplicity of coherent domains in chimera states. However, it has been shown for phase oscillator networks that time delay can also induce multi-chimeras. ${ }^{23}$ The existence of chimera states in systems with time-delayed couplings has been also reported in Refs. 50, 63-66. In particular, for coupled phase oscillator systems, it has been found that chimeras are robust to small time delays and delay distributions $^{64}$ and can become unstable depending on the value of delay. ${ }^{65}$ Here, we consider a model that includes not only phase but also amplitude dynamics and show how time delay in the coupling affects chimera states that exist in the undelayed system. We demonstrate that by varying the delay value, one can both conserve and eliminate chimera patterns.

Let us consider Eq. (2) modified by time-delayed coupling

$$
\begin{aligned}
\dot{u}_{k}(t)= & v_{k}(t) \\
\dot{v}_{k}(t)= & \varepsilon\left[1-u_{k}^{2}(t)\right] v_{k}(t)-u_{k}(t) \\
& +\frac{\sigma}{2 R} \sum_{j=k-R}^{k+R}\left\{b_{1}\left[u_{j}(t-\tau)-u_{k}(t)\right]\right. \\
& \left.+b_{2}\left[v_{j}(t-\tau)-v_{k}(t)\right]\right\}
\end{aligned}
$$

wth $k=1, \ldots, N$ modulo $N$, where $\tau$ is the delay time.
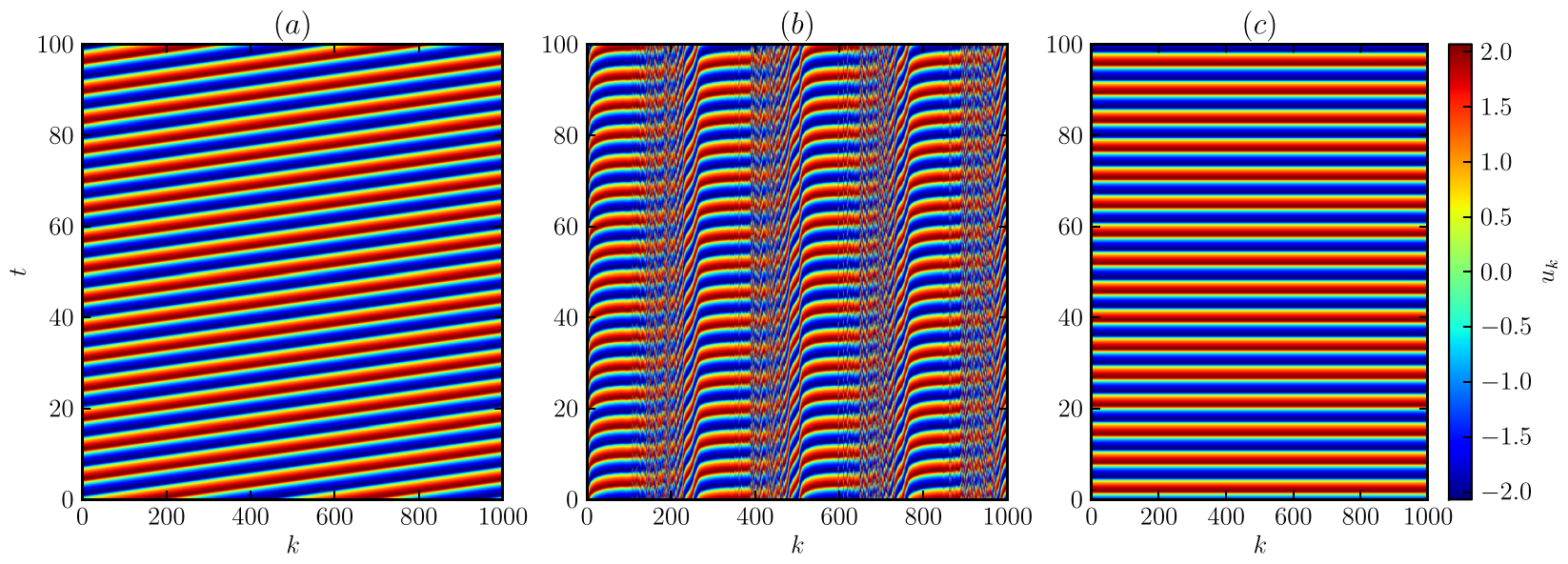

FIG. 6. Space-time plots of $u_{k}$ for different values of time delay: (a) $\tau=1$, (b) $\tau=3$, (c) $\tau=6$. Other parameters: $N=1000, b_{1}=1, b_{2}=0.1, r=0.4$, $\sigma=0.1, \varepsilon=1.5$. Initial conditions as shown in Fig. 5(a). Transients of 2000 time units are skipped. 
(a)

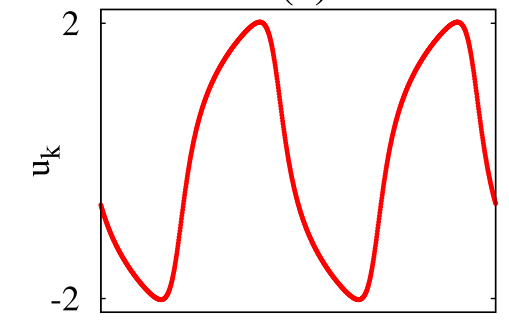

$-0.24$
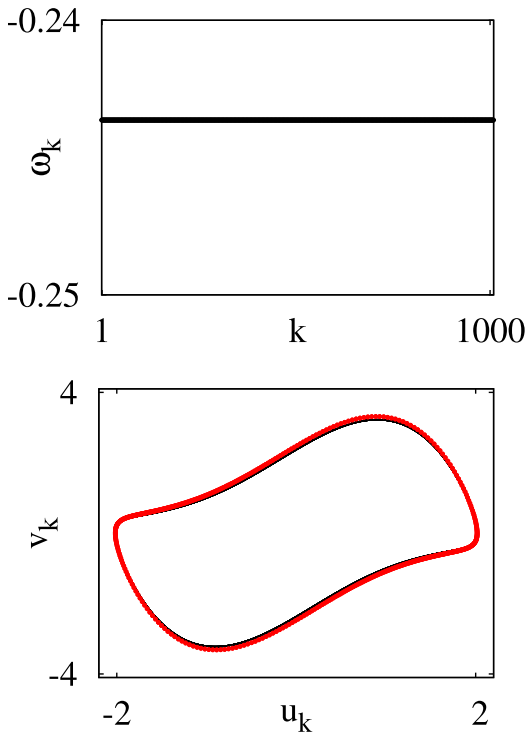

(b)

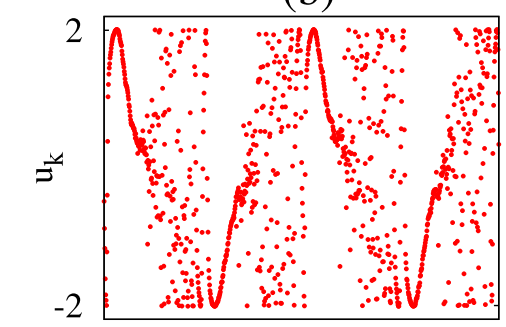

$-0.202$

$-0.205$
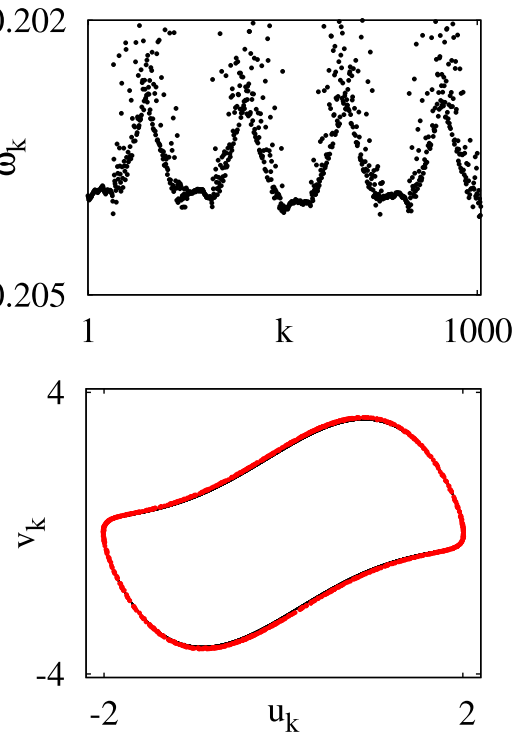

(c)
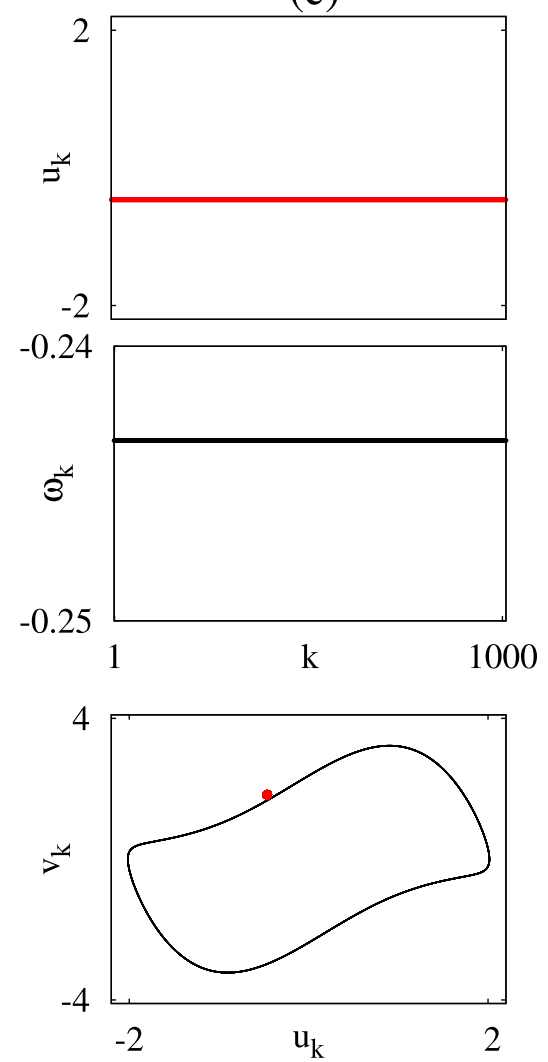

FIG. 7. Snapshots of the variables $u_{k}$ (upper panels), mean phase velocities $\omega_{k}$ (middle panels), and snapshots in the phase space ( $u_{k}, v_{k}$ ) (bottom panels, limit cycle of the uncoupled unit shown black). (a) $\tau=1$, (b) $\tau=3$, (c) $\tau=6$. Other parameters: $N=1000, b_{1}=1, b_{2}=0.1, r=0.4, \sigma=0.1, \varepsilon=1.5$.

Using the chimera state with four incoherent domains, shown in Fig. 5(a), as initial condition, i.e., as the history in the interval $[-\tau, 0]$, we fix all system parameters corresponding to this solution and show exemplary space-time patterns of Eq. (4) for different time delays. The period of a single uncoupled oscillator is close to $2 \pi$, and we neglect the transients of 2000 time units. For small time delay $(\tau=1)$, we observe a coherent traveling wave solution shown in Fig. $6(\mathrm{a})$. When the time delay is close to half the oscillation period $(\tau=3)$, the chimera pattern is stable and we continue to observe a chimera state with four incoherent domains as shown in Fig. 6(b). A larger time delay $(\tau=6)$, which is close to the period of a single oscillator, leads to complete synchronization of all oscillators, see Fig. 6(c). Figure 7 depicts snapshots of the variable $u_{k}$ (upper panels), the same snapshots in the phase plane $\left(u_{k}, v_{k}\right)$ together with the limit cycle of uncoupled oscillator, and the corresponding mean phase velocity profiles (middle panels), for the solutions shown in Fig. 6. The explanation for the effect of delay is that the delay time interacts with the intrinsic timescale (oscillation period) giving rise to resonance phenomena as found generally for delayed feedback control of steady states, deterministic limit cycles, and noise-induced oscillations, if the delay is an integer multiple or a half-integer multiple of the intrinsic timescale. ${ }^{67}$ Delay has a favorable effect on chimeras if $\tau$ is a half-integer multiple, and a favorable (stabilizing) effect on the synchronized oscillations if it is an integer multiple, and may induce traveling waves if it fits with neither condition. In case of the chimera (Fig. 7(b)), the delay leads to much longer transients, so that with the same length of time interval used for the calculation of the mean phase velocity as without delay, the profiles are more smeared out, but qualitatively similar.

Our numerical evidence shows similar results for other values of the bifurcation parameter $\varepsilon$. For chimera states with one or two incoherent domains and sinusoidal character of the oscillations, small delay can lead not only to traveling wave solutions but also to chimera states with higher number of incoherent domains.

These examples demonstrate that time delay introduced in the coupling can either suppress or preserve the chimera patterns depending upon the value of the delay time relative to the intrinsic oscillation period.

\section{CONCLUSION}

In the current study, we have demonstrated how the character of the local oscillator dynamics influences chimera states in networks of nonlocally coupled Van der Pol oscillators. Changing the bifurcation parameter of the single oscillators allows us to interpolate continuously between sinusoidal and strongly nonlinear relaxation oscillations. We have shown that nonlinearity facilitates multi-chimera states.

For small values of the bifurcation parameter $\varepsilon$ (sinusoidal oscillations), chimera states are characterized by lower multiplicity and more pronounced amplitude dynamics, and the maxima in the mean phase velocity profiles correspond to the coherent domains. Moving towards the relaxation 
oscillation regime, with increasing $\varepsilon$, leads to a higher multiplicity of chimera states, but weaker amplitude dynamics. In contrast to the previous case, the coherent domains correspond to the minima of the mean phase velocity profiles, i.e., the profiles are flipped. We have also found that time delay in the coupling strongly affects the chimera patterns in the system and can lead to chimera suppression and the formation of traveling waves and complete synchronization.

We have presented (multi-) chimera states of different types: (i) pure phase chimeras, which are similar to those found for Kuramoto phase oscillators or weakly coupled amplitude-phase models and (ii) amplitude-mediated chimeras with strong amplitude-phase coupling.

Our findings give new insight into the intriguing phenomena of chimera states and demonstrate that the character of the local dynamics has a strong influence on the chimera patterns in the whole network. These results could be useful from the point of view of applications dealing with different kinds of oscillators, as they can be realized, e.g., in electronic circuits.

\section{ACKNOWLEDGMENTS}

This work was supported by Deutsche Forschungsgemeinschaft in the framework of Collaborative Research Center SFB 910. P.H. acknowledges support by BMBF (grant no. 01Q1001B) in the framework of BCCN Berlin.

${ }^{1}$ A. Pikovsky, M. G. Rosenblum, and J. Kurths, Synchronization, A Universal Concept in Nonlinear Sciences (Cambridge University Press, Cambridge, 2001).

${ }^{2}$ S. Boccaletti, V. Latora, Y. Moreno, M. Chavez, and D. U. Hwang, Phys. Rep. 424, 175 (2006).

${ }^{3}$ Y. Kuramoto and D. Battogtokh, Nonlinear Phenom. Complex Syst. 5, 380 (2002).

${ }^{4}$ D. M. Abrams and S. H. Strogatz, Phys. Rev. Lett. 93, 174102 (2004).

${ }^{5}$ C. R. Laing, Physica D 238, 1569 (2009).

${ }^{6}$ A. E. Motter, Nat. Phys. 6, 164 (2010).

${ }^{7}$ E. A. Martens, C. R. Laing, and S. H. Strogatz, Phys. Rev. Lett. 104, 044101 (2010).

${ }^{8}$ O. E. Omel'chenko, M. Wolfrum, and Y. L. Maistrenko, Phys. Rev. E 81, 065201(R) (2010).

${ }^{9}$ O. E. Omel'chenko, M. Wolfrum, S. Yanchuk, Y. L. Maistrenko, and O. Sudakov, Phys. Rev. E 85, 036210 (2012).

${ }^{10}$ E. A. Martens, Chaos 20, 043122 (2010).

${ }^{11}$ M. Wolfrum, O. E. Omel'chenko, S. Yanchuk, and Y. L. Maistrenko, Chaos 21, 013112 (2011)

${ }^{12}$ T. Bountis, V. Kanas, J. Hizanidis, and A. Bezerianos, Eur. Phys. J. Spec. Top. 223, 721 (2014).

${ }^{13}$ M. J. Panaggio and D. M. Abrams, Nonlinearity 28, R67 (2015).

${ }^{14}$ I. Omelchenko, Y. L. Maistrenko, P. Hövel, and E. Schöll, Phys. Rev. Lett. 106, 234102 (2011).

${ }^{15}$ I. Omelchenko, B. Riemenschneider, P. Hövel, Y. L. Maistrenko, and E. Schöll, Phys. Rev. E 85, 026212 (2012).

${ }^{16}$ I. Omelchenko, O. E. Omel'chenko, P. Hövel, and E. Schöll, Phys. Rev. Lett. 110, 224101 (2013).

${ }^{17}$ J. Hizanidis, V. Kanas, A. Bezerianos, and T. Bountis, Int. J. Bifurcat. Chaos 24, 1450030 (2014).

${ }^{18}$ A. Vüllings, J. Hizanidis, I. Omelchenko, and P. Hövel, New J. Phys. 16, 123039 (2014).

${ }^{19}$ D. P. Rosin, D. Rontani, N. D. Haynes, E. Schöll, and D. J. Gauthier, Phys. Rev. E 90, 030902(R) (2014).

${ }^{20}$ S.-I. Shima and Y. Kuramoto, Phys. Rev. E 69, 036213 (2004).
${ }^{21}$ M. J. Panaggio and D. M. Abrams, Phys. Rev. Lett. 110, 094102 (2013).

${ }^{22}$ M. J. Panaggio and D. M. Abrams, Phys. Rev. E 91, 022909 (2015).

${ }^{23}$ G. C. Sethia, A. Sen, and F. M. Atay, Phys. Rev. Lett. 100, 144102 (2008).

${ }^{24}$ Yu. Maistrenko, A. Vasylenko, O. Sudakov, R. Levchenko, and V. Maistrenko, Int. J. Bifurcat. Chaos 24(8), 1440014 (2014).

${ }^{25}$ J. Xie, E. Knobloch, and H.-C. Kao, Phys. Rev. E 90, 022919 (2014).

${ }^{26}$ G. C. Sethia, A. Sen, and G. L. Johnston, Phys. Rev. E 88, 042917 (2013).

${ }^{27}$ G. C. Sethia and A. Sen, Phys. Rev. Lett. 112, 144101 (2014).

${ }^{28}$ A. Zakharova, M. Kapeller, and E. Schöll, Phys. Rev. Lett. 112, 154101 (2014).

${ }^{29}$ A. Yeldesbay, A. Pikovsky, and M. Rosenblum, Phys. Rev. Lett. 112, 144103 (2014).

${ }^{30}$ L. Schmidt and K. Krischer, Phys. Rev. Lett. 114, 034101 (2015).

${ }^{31}$ F. Böhm, A. Zakharova, E. Schöll, and K. Lüdge, Phys. Rev. E 91, 040901(R) (2015).

${ }^{32}$ T. W. Ko and G. B. Ermentrout, Phys. Rev. E 78, 016203 (2008).

${ }^{33}$ M. Shanahan, Chaos 20, 013108 (2010).

${ }^{34}$ C. R. Laing, K. Rajendran, and I. G. Kevrekidis, Chaos 22, 013132 (2012).

${ }^{35}$ N. Yao, Z.-G. Huang, Y.-C. Lai, and Z. Zheng, Sci. Rep. 3, 3522 (2013).

${ }^{36}$ Y. Zhu, Z. Zheng, and J. Yang, Phys. Rev. E 89, 022914 (2014).

${ }^{37}$ I. Omelchenko, A. Provata, J. Hizanidis, E. Schöll, and P. Hövel, Phys. Rev. E 91, 022917 (2015).

${ }^{38}$ A. Buscarino, M. Frasca, L. V. Gambuzza, and P. Hövel, Phys. Rev. E 91, 022817 (2015).

${ }^{39}$ C. R. Laing, Phys. Rev. E 81, 066221 (2010).

${ }^{40}$ N. C. Rattenborg, C. J. Amlaner, and S. L. Lima, Neurosci. Biobehav. Rev. 24, 817 (2000)

${ }^{41}$ C. R. Laing and C. C. Chow, Neural Comput. 13, 1473 (2001).

${ }^{42}$ H. Sakaguchi, Phys. Rev. E 73, 031907 (2006).

${ }^{43}$ A. E. Filatova, A. E. Hramov, A. A. Koronovskii, and S. Boccaletti, Chaos 18, 023133 (2008).

${ }^{44}$ J. C. Gonzalez-Avella, M. G. Cosenza, and M. S. Miguel, Physica A 399, 24 (2014)

${ }^{45}$ O. E. Omel'chenko, Nonlinearity 26, 2469 (2013).

${ }^{46}$ J. Sieber, O. E. Omel'chenko, and M. Wolfrum, Phys. Rev. Lett. 112, 054102 (2014).

${ }^{47}$ C. Bick and E. A. Martens, New J. Phys. 17, 033030 (2015).

${ }^{48}$ A. M. Hagerstrom, T. E. Murphy, R. Roy, P. Hövel, I. Omelchenko, and E. Schöll, Nat. Phys. 8, 658 (2012).

${ }^{49}$ M. R. Tinsley, S. Nkomo, and K. Showalter, Nat. Phys. 8, 662 (2012).

${ }^{50}$ S. Nkomo, M. R. Tinsley, and K. Showalter, Phys. Rev. Lett. 110, 244102 (2013).

${ }^{51}$ E. A. Martens, S. Thutupalli, A. Fourrière, and O. Hallatschek, Proc. Nat. Acad. Sci. U. S. A. 110, 10563 (2013).

${ }^{52}$ L. Larger, B. Penkovsky, and Y. L. Maistrenko, Phys. Rev. Lett. 111, 054103 (2013).

${ }^{53}$ L. V. Gambuzza, A. Buscarino, S. Chessari, L. Fortuna, R. Meucci, and M. Frasca, Phys. Rev. E 90, 032905 (2014).

${ }^{54}$ L. Schmidt, K. Schönleber, K. Krischer, and V. Garcia-Morales, Chaos 24, 013102 (2014).

${ }^{55} \mathrm{M}$. Wickramasinghe and I. Z. Kiss, PLoS ONE 8, e80586 (2013).

${ }^{56}$ E. A. Viktorov, T. Habruseva, S. P. Hegarty, G. Huyet, and B. Kelleher, Phys. Rev. Lett. 112, 224101 (2014).

${ }^{57}$ N. Lazarides, G. Neofotistos, and G. P. Tsironis, Phys. Rev. B 91, 054303 (2015).

${ }^{58}$ B. Van der Pol, Philos. Mag. 2, 978 (1926).

${ }^{59}$ A. Zakharova, A. Feoktistov, T. Vadivasova, and E. Schöll, Eur. Phys. J Spec. Top. 222, 2481-2495 (2013).

${ }^{60}$ L. A. Low, P. G. Reinhall, and D. W. Storti, J. Vibr. Acoust. 125, 162 (2003).

${ }^{61}$ L. A. Low, P. G. Reinhall, D. W. Storti, and E. B. Goldman, Struct. Control Health Monit. 13, 417-429 (2006).

${ }^{62}$ D. W. Storti and P. G. Reinhall, J. Vibr. Acous. 122, 318 (2000).

${ }^{63}$ O. E. Omel'chenko, Yu. L. Maistrenko, and P. A. Tass, Phys. Rev. Lett. 100, 044105 (2008).

${ }^{64}$ R. Ma, J. Wang, and Z. Liu, Europhys. Lett. 91, 40006 (2010).

${ }^{65}$ J. H. Sheeba, V. K. Chandrasekar, and M. Lakshmanan, Phys. Rev. E 79, 055203(R) (2009).

${ }^{66}$ J. H. Sheeba, V. K. Chandrasekar, and M. Lakshmanan, Phys. Rev. E 81, 046203 (2010).

${ }^{67}$ Handbook of Chaos Control, edited by E. Schöll and H. G. Schuster (Wiley-VCH, Weinheim, 2008). 